\title{
ERROR ANALYSIS OF DISCONTINUOUS GALERKIN METHODS FOR STOKES PROBLEM UNDER MINIMAL REGULARITY
}

\author{
S. BADIA, R. CODINA, T. GUDI, AND J. GUZMÁN
}

\begin{abstract}
In this article, we analyze several discontinuous Galerkin methods (DG) for the Stokes problem under the minimal regularity on the solution. We assume that the velocity $\mathbf{u}$ belongs to $\left[H_{0}^{1}(\Omega)\right]^{d}$ and the pressure $p \in L_{0}^{2}(\Omega)$. First, we analyze standard DG methods assuming that the right hand side $\mathbf{f}$ belongs to $\left[H^{-1}(\Omega) \cap L^{1}(\Omega)\right]^{d}$. A DG method that is well defined for $\mathbf{f}$ belonging to $\left[H^{-1}(\Omega)\right]^{d}$ is then investigated. The methods under study include stabilized DG methods using equal order spaces and inf-sup stable ones where the pressure space is one polynomial degree less than the velocity space.
\end{abstract}

\section{INTRODUCTION}

Let $\Omega \subset \mathbb{R}^{d}$ be a bounded polyhedral domain with boundary $\partial \Omega$. Let $\mathbf{V}=\left[H_{0}^{1}(\Omega)\right]^{d}$ and $Q=L_{0}^{2}(\Omega):=\left\{q \in L^{2}(\Omega):(q, 1)=0\right\}$. Here and throughout $(\cdot, \cdot)$ denotes the $L^{2}(\Omega)$ inner product. We also use the notation $(\cdot, \cdot)$ to denote the inner product on $\left[L^{2}(\Omega)\right]^{d}$.

For a given $\mathbf{f} \in\left[H^{-1}(\Omega)\right]^{d}$, the Stokes problem consists to find $[\mathbf{u}, p] \in \mathbf{V} \times Q$ such that

$$
\begin{aligned}
a(\mathbf{u}, \mathbf{v})+b(\mathbf{v}, p) & =\mathbf{f}(\mathbf{v}) & & \forall \mathbf{v} \in \mathbf{V}, \\
b(\mathbf{u}, q) & =0 & & \forall q \in Q,
\end{aligned}
$$

where

$$
\begin{aligned}
a(\mathbf{w}, \mathbf{v}) & =(\nabla \mathbf{w}, \nabla \mathbf{v}) \quad \forall \mathbf{w}, \mathbf{v} \in \mathbf{V}, \\
b(\mathbf{v}, q) & =-(\nabla \cdot \mathbf{v}, q) \quad \forall \mathbf{v} \in \mathbf{V}, q \in Q .
\end{aligned}
$$

The space $\mathbf{V}$ is endowed with the norm $\|\cdot\|_{\mathbf{v}}$ defined by $\|\mathbf{v}\|_{\mathbf{v}}=(\nabla \mathbf{v}, \nabla \mathbf{v})^{1 / 2}$.

Existence and uniqueness of the velocity $\mathbf{u}$ follows from the Lax-Milgram lemma in the space $\mathbf{Z}=\{\mathbf{v} \in \mathbf{V}: b(\mathbf{v}, q)=0 \quad \forall q \in Q\}$. Existence of a pressure is obtained by the well-known inf-sup condition

$$
\beta\|q\|_{L^{2}(\Omega)} \leq \sup _{\mathbf{v} \in \mathbf{V}, \mathbf{v} \neq 0} \frac{b(\mathbf{v}, q)}{\|\mathbf{v}\|_{\mathbf{v}}} \quad \forall q \in Q .
$$

The discrete analog of the inf-sup condition leads to stable numerical methods; see for example [6]. However, in order to satisfy the discrete inf-sup condition the discrete pressure and velocity spaces are dependent on each other. For example, as is well known, equal

1991 Mathematics Subject Classification. 65N30, 65N15.

Key words and phrases. error estimates, finite element, discontinuous Galerkin, stokes problems, stabilized methods. 
order spaces are not inf-sup stable. On the other hand, stabilized methods allows one to use discrete spaces that are not inf-sup stable.

There are now many DG methods for Stokes' flow using the velocity-pressure formulation; see for example [24, 12, 21]. Error analysis have been performed for most DG methods in the literature. However, they often assume sufficient regularity of the exact solution. In this paper we perform an error analysis of some DG methods for the Stokes problem where we only assume minimal regularity $[\mathbf{u}, p] \in \mathbf{V} \times Q$. Moreover, we assume $\mathbf{f} \in\left[H^{-1}(\Omega) \cap L^{1}(\Omega)\right]^{d}$. We note that DG methods are not well-defined for functions $\mathbf{f}$ only belonging to $\left[H^{-1}(\Omega)\right]^{d}$ since DG test functions do not belong to $\left[H^{1}(\Omega)\right]^{d}$. However, we show how to modify the right hand side of the DG method so that we can define a method for $\mathbf{f} \in\left[H^{-1}(\Omega)\right]^{d}$. The approach we take in this paper is the one used by Gudi [19, 20] for elliptic problems where residual estimates are used in the analysis.

Di Pietro and Ern [17] were the first to address convergence of DG methods for problems with minimal regularity. There they considered solutions $[\mathbf{u}, p] \in \mathbf{V} \times Q$ and $\mathbf{f} \in\left[L^{p}(\Omega)\right]^{d}$ for $p>1$ when $d=2$ and $p \geq 6 / 5$ when $d=3$ (these restrictions are due to Sobolev embeddings). Their approach was to use discrete compactness arguments. Shortly after, Gudi [19] considered elliptic problems where he assumed the solution $u$ to be in $H_{0}^{1}(\Omega)$ and proved error estimates which give rates of convergence in the case of smoothness. There he assumed that the right hand side belongs to $L^{2}(\Omega)$. Finally, Rivière and proved optimal error estimates for the Laplace problem in two dimensions using $W^{2, p}$ elliptic regularity for $p>1$.

As mentioned above, we will apply the techniques in [19] to give error estimates for the Stokes problem. Here we show, following the argument of Gudi [19], that if one is a bit more careful one only needs data belonging to $\left[H^{-1}(\Omega) \cap L^{1}(\Omega)\right]^{d}$. Our estimates will depend on an oscillation term measuring the sum of local $H^{-1}$ norms of the difference between $\mathbf{f}$ and its $L^{2}$ projection onto piecewise polynomials; see Theorem 3.1. Similar oscillation terms were considered by Cohen et al. [15] when dealing with data in $\left[H^{-1}(\Omega)\right]^{d}$. Using Sobolev embeddings we can prove convergence results under the assumptions used in [17] while also showing convergence rates when slightly more regular data is used. We stress that our a-priori analysis does not depend on elliptic regularity.

Pressure estimates require special care. When the pressure polynomial space is one degree less than the velocity space, one only needs to use that the discrete velocity (or the $H$ (div; $\Omega$ ) conforming part of the space) and pressure space form a $H(\operatorname{div} ; \Omega) \times L^{2}(\Omega)$ stable pair (such ideas were used for example in [24]). In the equal order case, one needs to bound also the higher modes of the pressure approximation. We do this by estimating the traces of the error on faces of the simplicial decomposition. In the equal order case we consider two distinct methods, one which penalizes the jumps of the pressure (see for example [24]) and a method introduced in [14] and applied to DG methods in [13] that penalizes the jumps of the total flux. Since penalizing the jumps of the pressure is inconsistent with pressure only belonging to $L^{2}(\Omega)$ we get best approximation results in the space $Q_{h} \cap H^{1}(\Omega)$, where $Q_{h}$ is the DG discrete space for the pressure. On the other hand when we use the DG method in [13] we get best approximation in the space $Q_{h}$. 
Since standard DG methods are not well defined for $\mathbf{f} \in\left[H^{-1}(\Omega)\right]^{d}$ we modify the righthand side in order to consider this case with the help of enrichment operators. We show that the proposed method converges strongly in $\left[H^{1}(\Omega)\right]^{d} \times L^{2}(\Omega)$. Moreover, we show that if one modifies the right-hand side appropriately (i.e. chooses the enrichment operator appropriately), a best approximation property will hold.

All our analysis is based on the assumption that the polynomial degree of the finite element approximation is kept constant. Thus, our results proving convergence under minimal regularity assumptions hold in the case in which the mesh size tends to zero.

The paper is organized as follows. In Section 2 we introduce notation and some preliminary results. We propose the methods to be analyzed in Section 3 and prove velocity error estimates. Section 4 is devoted to pressure error estimates. The analysis for a method that penalizes flux jumps is included in Section 5. Finally, the extension of the previous analyses to forcing terms in $\left[H^{-1}(\Omega)\right]^{d}$ is carried out in Section 6.

\section{Notation AND PRELIminaries}

The following notation will be used throughout the article:

$\mathcal{T}_{h}=$ face to face, shape regular simplicial triangulations of $\Omega$

$T=$ a simplex of $\mathcal{T}_{h} \quad h_{T}=$ diameter of $T \quad h=\max \left\{h_{T}: T \in \mathcal{T}_{h}\right\}$

$\mathcal{V}_{h}^{i}=$ set of all vertices in $\mathcal{T}_{h}$ that are in $\Omega$

$\mathcal{V}_{h}^{b}=$ set of all vertices in $\mathcal{T}_{h}$ that are on $\partial \Omega$

$\mathcal{V}_{h}=\mathcal{V}^{i} \cup \mathcal{V}^{b}$

$\mathcal{E}_{h}^{i}=$ set of all interior edges of $\mathcal{T}_{h}$

$\mathcal{E}_{h}^{b}=$ set of all boundary edges of $\mathcal{T}_{h}$

$\mathcal{E}_{h}=\mathcal{E}_{h}^{i} \cup \mathcal{E}_{h}^{b}$

$h_{e}=|e|$, the diameter of $e \in \mathcal{E}_{h}$

$\mathcal{T}_{z}=$ set of all simplices sharing the vertex $z$

$\mathcal{T}_{e}=$ patch of two simplices sharing the face $e$

$\nabla_{h}=$ piecewise (element-wise) gradient

$\mathbb{P}_{m}(T)=$ space of polynomials of degree less than or equal to $m \geq 0$ and defined on $T$

$I=$ Identity matrix of size $d \times d$.

Let us define the discrete spaces

$$
\mathbf{V}_{h}=\left\{\mathbf{v}_{h} \in\left[L^{2}(\Omega)\right]^{d}:\left.\mathbf{v}_{h}\right|_{T} \in\left[\mathbb{P}_{r}(T)\right]^{d}\right\} \quad \text { and } \quad Q_{h}^{s}=\left\{q_{h} \in Q:\left.q_{h}\right|_{T} \in \mathbb{P}_{s}(T)\right\} .
$$

We will consider the cases $s=r-1$ and the equal order case $s=r$. For the sake of convenience, let us define a broken Sobolev space

$$
H^{1}\left(\Omega, \mathcal{T}_{h}\right)=\left\{v \in L^{2}(\Omega):\left.v\right|_{T} \in H^{1}(T) \quad \forall T \in \mathcal{T}_{h}\right\} .
$$


In the problem setting, we require jump and mean definitions of discontinuous functions, vector functions and tensors. For any $e \in \mathcal{E}_{h}^{i}$, there are two simplices $T_{+}$and $T_{-}$such that $e=\partial T_{+} \cap \partial T_{-}$. Let $\mathbf{n}_{+}$be the unit normal of $e$ pointing from $T_{+}$to $T_{-}$, and $\mathbf{n}_{-}=-\mathbf{n}_{+}$. For any $v \in H^{1}\left(\Omega, \mathcal{T}_{h}\right)$, we define the jump and mean of $v$ on $e$ by

$$
\llbracket v \rrbracket=v_{+} \mathbf{n}_{+}+v_{-} \mathbf{n}_{-}, \text {and }\{\{v\}\}=\frac{1}{2}\left(v_{+}+v_{-}\right) \text {, respectively, }
$$

where $v_{ \pm}=\left.v\right|_{T_{ \pm}}$. We define for $\mathbf{v} \in\left[H^{1}\left(\Omega, \mathcal{T}_{h}\right)\right]^{d}$ the jump and mean of $\mathbf{v}$ on $e \in \mathcal{E}_{h}^{i}$ by

$$
\llbracket \mathbf{v} \rrbracket=\mathbf{v}_{+} \cdot \mathbf{n}_{+}+\mathbf{v}_{-} \cdot \mathbf{n}_{-}, \text {and }\{\{\mathbf{v}\}\}=\frac{1}{2}\left(\mathbf{v}_{+}+\mathbf{v}_{-}\right) \text {, respectively. }
$$

We also require the full jump of vector valued functions. For $\mathbf{v} \in\left[H^{1}\left(\Omega, \mathcal{T}_{h}\right)\right]^{d}$, we define the full jump by

$$
\llbracket \underline{\mathbf{v}} \rrbracket=\mathbf{v}_{+} \otimes \mathbf{n}_{+}+\mathbf{v}_{-} \otimes \mathbf{n}_{-},
$$

where for two vectors in Cartesian coordinates $\mathbf{a}=\left(a_{i}\right)$ and $\mathbf{b}=\left(b_{j}\right)$, we define the matrix $\mathbf{a} \otimes \mathbf{b}=\left[a_{i} b_{j}\right]_{1 \leq i, j \leq d}$. Similarly, for tensors $\tau \in\left[H^{1}\left(\Omega, \mathcal{T}_{h}\right)\right]^{d \times d}$ the jump and mean on $e \in \mathcal{E}_{h}^{i}$ are defined by

$$
\llbracket \tau \rrbracket=\tau_{+} \mathbf{n}_{+}+\tau_{-} \mathbf{n}_{-}, \text {and }\{\{\tau\}\}=\frac{1}{2}\left(\tau_{+}+\tau_{-}\right) \text {, respectively. }
$$

For notational convenience, we also define the jump and mean on the boundary faces $e \in \mathcal{E}_{h}^{b}$ by modifying them appropriately. We use the definition of jump by understanding that $v_{-}=0$ (similarly, $\mathbf{v}_{-}=0$ and $\tau_{-}=0$ ) and the definition of mean by understanding that $v_{-}=v_{+}$(similarly, $\mathbf{v}_{-}=\mathbf{v}_{+}$and $\left.\tau_{-}=\tau_{+}\right)$.

In the analysis of next sections, we require the existence of an enriching operator $E_{h}$ : $\mathbf{V}_{h} \rightarrow \mathbf{V}_{h} \cap H_{0}^{1}(\Omega)$ such that

$$
\left(\sum_{T \in \mathcal{T}_{h}} h_{T}^{-2}\left\|E_{h} \mathbf{v}_{h}-\mathbf{v}_{h}\right\|_{L^{2}(T)}^{2}\right)^{1 / 2}+\left\|\nabla_{h}\left(E_{h} \mathbf{v}_{h}-\mathbf{v}_{h}\right)\right\|_{L^{2}(\Omega)} \leq C\left\|\mathbf{v}_{h}\right\|_{h},
$$

where

$$
\left\|\mathbf{v}_{h}\right\|_{h}^{2}=\sum_{T \in \mathcal{T}_{h}}\left\|\nabla \mathbf{v}_{h}\right\|_{L^{2}(T)}^{2}+\sum_{e \in \mathcal{E}_{h}^{i}} \int_{e} \frac{1}{h_{e}} \llbracket \underline{\llbracket \mathbf{v}_{h}} \rrbracket^{2} .
$$

It is well known that this type of enriching operators can be constructed by averaging techniques $[7,8]$.

We will also need the following inverse estimates [10].

Lemma 2.1. There exists a constant $C_{m}$ such that for all $v \in \mathbb{P}_{m}(T)$ one has

$$
\|v\|_{H^{1}(T)} \leq C_{m} h_{T}^{-1}\|v\|_{L^{2}(T)}
$$

and

$$
\|v\|_{L^{2}(\partial T)} \leq C_{m} h_{T}^{-1 / 2}\|v\|_{L^{2}(T)}
$$


For the next lemma we need to recall the definition of the $H^{-1}(D)$ norm:

$$
\|\mathbf{f}\|_{H^{-1}(D)}=\sup _{\mathbf{w} \in\left[H_{0}^{1}(D)\right]^{d}} \frac{\mathbf{f}(\mathbf{w})}{\|\mathbf{w}\|_{H^{1}(D)}} .
$$

For subsequent analyses, we define the restrictions of $\mathbf{f}$ to $H_{0}^{1}(T)$ by $\mathbf{f}_{T}$ and to $H_{0}^{1}\left(\mathcal{T}_{e}\right)$ by $\mathbf{f}_{e}$. That is

$$
\begin{aligned}
\mathbf{f}_{T}\left(\mathbf{v}_{1}\right)=\mathbf{f}\left(\tilde{\mathbf{v}}_{1}\right) & \forall \mathbf{v}_{1} \in H_{0}^{1}(T) \\
\mathbf{f}_{e}\left(\mathbf{v}_{2}\right)=\mathbf{f}\left(\tilde{\mathbf{v}}_{2}\right) & \forall \mathbf{v}_{2} \in H_{0}^{1}\left(\mathcal{T}_{e}\right),
\end{aligned}
$$

where $\tilde{\mathbf{v}}_{1}$ (or $\left.\tilde{\mathbf{v}}_{2}\right)$ is the extensions of $\mathbf{v}_{1}\left(\right.$ or $\left.\mathbf{v}_{2}\right)$ by zero outside of $T$ (or $\mathcal{T}_{e}$ ).

The following residual estimates which resemble local-efficiency estimates will be crucial for forthcoming analysis:

Lemma 2.2. Let $\mathbf{f}_{h} \in \mathbf{V}_{h}, \mathbf{v}_{h} \in \mathbf{V}_{h}$ and $q_{h} \in Q_{h}$ be arbitrary. Then, it holds that

$$
\begin{aligned}
h_{T}\left\|\mathbf{f}_{h}+\Delta \mathbf{v}_{h}-\nabla q_{h}\right\|_{L^{2}(T)} & \leq C\left(\left\|\nabla\left(\mathbf{u}-\mathbf{v}_{h}\right)\right\|_{L^{2}(T)}+\left\|p-q_{h}\right\|_{L^{2}(T)}+\left\|\mathbf{f}_{T}-\mathbf{f}_{h}\right\|_{H^{-1}(T)}\right), \\
\left\|h_{e}^{1 / 2} \llbracket \nabla_{h} \mathbf{v}_{h}-q_{h} I \rrbracket\right\|_{L^{2}(e)} & \leq C\left(\left\|\nabla_{h}\left(\mathbf{u}-\mathbf{v}_{h}\right)\right\|_{L^{2}\left(\mathcal{T}_{e}\right)}+\left\|p-q_{h}\right\|_{L^{2}\left(\mathcal{T}_{e}\right)}+\left\|\mathbf{f}_{e}-\mathbf{f}_{h}\right\|_{H^{-1}\left(\mathcal{T}_{e}\right)}\right) .
\end{aligned}
$$

Proof. We begin by proving the first estimate. Let $b_{T} \in H_{0}^{1}(T)$ be the polynomial bubble function that takes unit value at the barycenter of $T$. Then it is obvious that

$$
\left\|b_{T}\left(\mathbf{f}_{h}+\Delta \mathbf{v}_{h}-\nabla q_{h}\right)\right\|_{L^{2}(T)} \leq\left\|\mathbf{f}_{h}+\Delta \mathbf{v}_{h}-\nabla q_{h}\right\|_{L^{2}(T)},
$$

where $\mathbf{f}_{h} \in \mathbf{V}_{h}$ is an arbitrary polynomial on $T$. Using the fact that all norms are equivalent on a finite dimensional space and a scaling argument, there exists a positive constant $C_{1}$ such that

$$
C_{1}\left\|\mathbf{f}_{h}+\Delta \mathbf{v}_{h}-\nabla q_{h}\right\|_{L^{2}(T)}^{2} \leq\left\|b_{T}^{1 / 2}\left(\mathbf{f}_{h}+\Delta \mathbf{v}_{h}-\nabla q_{h}\right)\right\|_{L^{2}(T)}^{2} .
$$

The constant $C_{1}$ depends on the shape of $T$ and the polynomial order, but not on the diameter of $T$. Let $\mathbf{w}_{h}=b_{T}\left(\mathbf{f}_{h}+\Delta \mathbf{v}_{h}-\nabla q_{h}\right)$. Clearly $\mathbf{w}_{h} \in\left[H_{0}^{1}(T)\right]^{d}$. Then

$$
\begin{aligned}
C_{1} \int_{T}\left(\mathbf{f}_{h}+\Delta \mathbf{v}_{h}-\nabla q_{h}\right)^{2} & \leq \int_{T} \mathbf{w}_{h} \cdot\left(\mathbf{f}_{h}+\Delta \mathbf{v}_{h}-\nabla q_{h}\right) \\
& =\mathbf{f}_{T}\left(\mathbf{w}_{h}\right)+\int_{T} \mathbf{w}_{h} \cdot\left(\Delta \mathbf{v}_{h}-\nabla q_{h}\right)+\left(\mathbf{f}_{h}-\mathbf{f}_{T}\right)\left(\mathbf{w}_{h}\right),
\end{aligned}
$$

where $\mathbf{f}_{h}\left(\mathbf{w}_{h}\right)=\int_{T} \mathbf{f}_{h} \cdot \mathbf{w}_{h}$. Let $\tilde{\mathbf{w}}_{h}$ be the extension of $\mathbf{w}_{h}$ by zero outside of $T$. Then using (2.5), (1.1) and integration by parts,

$$
\begin{aligned}
\mathbf{f}_{T}\left(\mathbf{w}_{h}\right)+\int_{T} \mathbf{w}_{h} \cdot\left(\Delta \mathbf{v}_{h}-\nabla q_{h}\right)= & \mathbf{f}\left(\tilde{\mathbf{w}}_{h}\right)+\int_{T} \mathbf{w}_{h} \cdot\left(\Delta \mathbf{v}_{h}-\nabla q_{h}\right) \\
= & \int_{\Omega} \nabla \mathbf{u}: \nabla \tilde{\mathbf{w}}_{h}-\int_{\Omega} \nabla \cdot \tilde{\mathbf{w}}_{h} p \\
& -\int_{T} \nabla \mathbf{v}_{h}: \nabla \mathbf{w}_{h}+\int_{T} \nabla \cdot \mathbf{w}_{h} q_{h}
\end{aligned}
$$




$$
=\int_{T} \nabla\left(\mathbf{u}-\mathbf{v}_{h}\right): \nabla \mathbf{w}_{h}-\int_{T} \nabla \cdot \mathbf{w}_{h}\left(p-q_{h}\right) .
$$

Therefore

$$
\begin{aligned}
C_{1} h_{T}^{2}\left\|\mathbf{f}_{h}+\Delta \mathbf{v}_{h}-\nabla q_{h}\right\|_{L^{2}(T)}^{2} & \leq h_{T}^{2}\left\|\nabla\left(\mathbf{u}-\mathbf{v}_{h}\right)\right\|_{L^{2}(T)}\left\|\nabla \mathbf{w}_{h}\right\|_{L^{2}(T)} \\
& +h_{T}^{2}\left\|\nabla \cdot \mathbf{w}_{h}\right\|_{L^{2}(T)}\left\|p-q_{h}\right\|_{L^{2}(T)}+h_{T}^{2}\left\|\mathbf{w}_{h}\right\|_{H^{1}(T)}\left\|\mathbf{f}_{T}-\mathbf{f}_{h}\right\|_{H^{-1}(T)} .
\end{aligned}
$$

Using an inverse inequality,

$$
C h_{T}\left\|\mathbf{f}_{h}+\Delta \mathbf{v}_{h}-\nabla q_{h}\right\|_{L^{2}(T)} \leq\left\|\nabla\left(\mathbf{u}-\mathbf{v}_{h}\right)\right\|_{L^{2}(T)}+\left\|p-q_{h}\right\|_{L^{2}(T)}+\left\|\mathbf{f}_{T}-\mathbf{f}_{h}\right\|_{H^{-1}(T)} .
$$

It completes the proof of the first inequality.

To prove the second inequality, let $b_{e} \in H_{0}^{1}\left(\mathcal{T}_{e}\right)$ be the face bubble function that takes unit value at the barycenter of the face $e$. Let $\psi$ be the extension of $\llbracket \nabla_{h} \mathbf{v}_{h}-q_{h} I \rrbracket$ to $\mathcal{T}_{e}$ by constants along the lines orthogonal to the edge $e$. Let $\mathbf{w}_{h}=b_{e} \psi$. Then by scaling

$$
\left\|\mathbf{w}_{h}\right\|_{L^{2}\left(\mathcal{T}_{e}\right)} \leq C\left\|h_{e}^{1 / 2} \llbracket \nabla_{h} \mathbf{v}_{h}-q_{h} I \rrbracket\right\|_{L^{2}(e)} .
$$

Using again a scaling argument,

$$
\begin{aligned}
C\left\|\llbracket \nabla_{h} \mathbf{v}_{h}-q_{h} I \rrbracket\right\|_{L^{2}(e)}^{2} \leq & \left\|b_{e}^{1 / 2} \llbracket \nabla_{h} \mathbf{v}_{h}-q_{h} I \rrbracket\right\|_{L^{2}(e)}^{2}=\int_{e} \mathbf{w}_{h} \cdot \llbracket \nabla_{h} \mathbf{v}_{h}-q_{h} I \rrbracket \\
= & \sum_{T \subset \mathcal{T}_{e}} \int_{T} \nabla \mathbf{v}_{h}: \nabla \mathbf{w}_{h}-\sum_{T \subset \mathcal{T}_{e}} \int_{T} \nabla \cdot \mathbf{w}_{h} q_{h} \\
& +\sum_{T \subset \mathcal{T}_{e}} \int_{T}\left(\Delta \mathbf{v}_{h}-\nabla q_{h}\right) \cdot \mathbf{w}_{h} \\
= & \sum_{T \subset \mathcal{T}_{e}} \int_{T} \nabla\left(\mathbf{v}_{h}-\mathbf{u}\right): \nabla \mathbf{w}_{h}-\sum_{T \subset \mathcal{T}_{e}} \int_{T} \nabla \cdot \mathbf{w}_{h}\left(q_{h}-p\right) \\
& +\sum_{T \subset \mathcal{T}_{e}} \int_{T}\left(\Delta \mathbf{v}_{h}-\nabla q_{h}+\mathbf{f}_{h}\right) \cdot \mathbf{w}_{h}+\left(\mathbf{f}_{e}-\mathbf{f}_{h}\right)\left(\mathbf{w}_{h}\right),
\end{aligned}
$$

where $\mathbf{f}_{h}\left(\mathbf{w}_{h}\right)=\int_{\mathcal{T}_{e}} \mathbf{f}_{h} \cdot \mathbf{w}_{h}$. Using the Cauchy-Schwarz inequality and an inverse inequality,

$$
\begin{aligned}
h_{e}\|\| \nabla_{h} \mathbf{v}_{h}-q_{h} I \rrbracket \|_{L^{2}(e)}^{2} \leq & C\left(\left\|\nabla_{h}\left(\mathbf{v}_{h}-\mathbf{u}\right)\right\|_{L^{2}\left(\mathcal{T}_{e}\right)}+\left\|p-q_{h}\right\|_{L^{2}\left(\mathcal{T}_{e}\right)}\right)\left\|\mathbf{w}_{h}\right\|_{L^{2}\left(\mathcal{T}_{e}\right)} \\
& +C\left(\sum_{T \subset \mathcal{T}_{e}} h_{T}^{2}\left\|\Delta \mathbf{v}_{h}-\nabla q_{h}+\mathbf{f}_{h}\right\|_{L^{2}(T)}^{2}\right)^{1 / 2}\left\|\mathbf{w}_{h}\right\|_{L^{2}\left(\mathcal{T}_{e}\right)} \\
& +C\left\|\mathbf{f}_{e}-\mathbf{f}_{h}\right\|_{H^{-1}\left(\mathcal{T}_{e}\right)}\left\|\mathbf{w}_{h}\right\|_{L^{2}\left(\mathcal{T}_{e}\right)} .
\end{aligned}
$$

Using (2.7) and the first inequality of this lemma, we complete the proof. 


\section{Discontinuous Galerkin Methods}

We now describe the DG methods we will consider. For simplicity we only consider the symmetric interior penalty formulation for the Laplace operator (see the form $\mathcal{A}_{h}$ below), but the analysis can readily be applied to other methods found in [3]. Define

$$
\begin{aligned}
\mathcal{A}_{h}\left(\mathbf{w}_{h}, \mathbf{v}_{h}\right)= & \sum_{T \in \mathcal{T}_{h}} \int_{T} \nabla \mathbf{w}_{h}: \nabla \mathbf{v}_{h}-\sum_{e \in \mathcal{E}_{h}} \int_{e}\left\{\left\{\nabla_{h} \mathbf{w}_{h}\right\}\right\}: \llbracket \underline{\mathbf{v}_{h}} \rrbracket \\
& -\sum_{e \in \mathcal{E}_{h}} \int_{e}\left\{\left\{\nabla_{h} \mathbf{v}_{h}\right\}\right\}: \llbracket \underline{\llbracket \mathbf{w}_{h} \rrbracket}+\sum_{e \in \mathcal{E}_{h}} \int_{e} \frac{\eta}{h_{e}} \llbracket \underline{\mathbf{w}_{h} \rrbracket} \rrbracket: \llbracket \underline{\mathbf{v}_{h}} \rrbracket,
\end{aligned}
$$

where $\eta$ is a stabilization (algorithmic) parameter, and

$$
\mathcal{B}_{h}\left(\mathbf{v}_{h}, q_{h}\right)=-\sum_{T \in \mathcal{T}_{h}} \int_{T} \nabla \cdot \mathbf{v}_{h} q_{h}+\sum_{e \in \mathcal{E}_{h}} \int_{e}\left\{\left\{q_{h}\right\}\right\} \llbracket \mathbf{v}_{h} \rrbracket .
$$

The DG method we consider is to find $\left[\mathbf{u}_{h}, p_{h}\right] \in \mathbf{V}_{h} \times Q_{h}$ such that

$$
\begin{aligned}
\mathcal{A}_{h}\left(\mathbf{u}_{h}, \mathbf{v}_{h}\right)+\mathcal{B}_{h}\left(\mathbf{v}_{h}, p_{h}\right) & =\mathbf{f}\left(\mathbf{v}_{h}\right) & & \forall \mathbf{v}_{h} \in \mathbf{V}_{h}, \\
\mathcal{B}_{h}\left(\mathbf{u}_{h}, q_{h}\right)-\mathcal{S}_{h}\left(p_{h}, q_{h}\right) & =0 & & \forall q_{h} \in Q_{h},
\end{aligned}
$$

where $\mathcal{S}_{h}$ is a semi-positive definite bilinear form, i.e., $\mathcal{S}_{h}\left(q_{h}, q_{h}\right) \geq 0$ for all $q_{h} \in Q_{h}$. In the case $Q_{h}=Q_{h}^{r-1}$ we set $\mathcal{S}_{h} \equiv 0$. In the case $Q_{h}=Q_{h}^{r}$ (i.e. equal order case) we set

$$
\mathcal{S}_{h}(q, p)=\sum_{e \in \mathcal{E}_{h}^{i}} h_{e} \int_{e} \llbracket q \rrbracket \cdot \llbracket p \rrbracket .
$$

The reason why extra stabilization is needed for the equal order case is to control the higher modes of the approximate pressure. In a later section we describe the method were we penalize the total flux. The error estimates will be slightly different.

Note that we are implicitly assuming that $\mathbf{f}\left(\mathbf{v}_{h}\right)$ makes sense for all $\mathbf{v}_{h} \in \mathbf{V}_{h}$. This is certainly not the case if we only assume that $\mathbf{f} \in\left[H^{-1}(\Omega)\right]^{d}$. From now on we assume that $\mathbf{f} \in\left[H^{-1}(\Omega) \cap L^{1}(\Omega)\right]^{d}$.

For the rest of the analysis, we define $\mathbf{Z}_{h}=\left\{\mathbf{v}_{h} \in \mathbf{V}_{h}: \mathcal{B}_{h}\left(\mathbf{v}_{h}, q_{h}\right)=0 \quad \forall q_{h} \in Q_{h}\right\}$. We note that $\Pi^{\mathrm{RT}} \mathbf{u}$ belongs to $\mathbf{Z}_{h}$ where $\Pi^{\mathrm{RT}}:\left[H_{0}^{1}(\Omega)\right]^{d} \rightarrow \mathbf{V}_{h} \cap H_{0}(\operatorname{div} ; \Omega)$ is the RaviartThomas projection of index $r$; see for example $[22]$. Therefore, if $\mathbf{u} \in\left[H^{1+s}(\Omega)\right]^{d}$ for any $s \geq 0$ we have

$$
\inf _{\mathbf{v}_{h} \in \mathbf{Z}_{h}}\left\|\mathbf{u}-\mathbf{v}_{h}\right\|_{h} \leq C\left\|\mathbf{u}-\Pi^{\mathrm{RT}} \mathbf{u}\right\|_{h} \leq C h^{s}\|\mathbf{u}\|_{H^{1+s}(\Omega)} .
$$

We also need the following coercivity estimate. For a sufficiently large stabilizing parameter $\eta>0$, it holds that

$$
C\left\|\mathbf{v}_{h}\right\|_{h}^{2} \leq \mathcal{A}_{h}\left(\mathbf{v}_{h}, \mathbf{v}_{h}\right) \quad \forall \mathbf{v}_{h} \in \mathbf{V}_{h}
$$


In order to describe the error for the velocity we need to define $O \operatorname{sc}(\mathbf{f}, \Omega)$. Let $P$ : $\left[L^{1}(\Omega)\right]^{d} \rightarrow \mathbf{V}_{h}$ be the $L^{2}$ orthogonal projection onto $\mathbf{V}_{h}$. That is,

$$
\int_{\Omega}(P \mathbf{f}-\mathbf{f}) \cdot \mathbf{w}=0 \quad \mathbf{w} \in \mathbf{V}_{h} .
$$

Note that this is well defined for functions in $\mathbf{f} \in\left[L^{1}(\Omega)\right]^{d}$. Let us also define

$$
\operatorname{Osc}(\mathbf{f}, \Omega):=\left(\sum_{e \in \mathcal{E}_{h}}\left\|\mathbf{f}_{e}-P \mathbf{f}\right\|_{H^{-1}\left(\mathcal{T}_{e}\right)}^{2}\right)^{1 / 2}
$$

The following obvious estimate is useful to note for the subsequent analysis:

$$
\sum_{T \subset \mathcal{T}_{e}}\left\|\mathbf{f}_{T}-P \mathbf{f}\right\|_{H^{-1}(T)}^{2} \leq C\left\|\mathbf{f}_{e}-P \mathbf{f}\right\|_{H^{-1}\left(\mathcal{T}_{e}\right)}^{2} .
$$

We can now prove an estimate for the velocity.

Theorem 3.1. It holds that

$$
\begin{aligned}
\left\|\mathbf{u}-\mathbf{u}_{h}\right\|_{h}+\mathcal{S}_{h}\left(p_{h}, p_{h}\right)^{1 / 2} \leq & C\left(\inf _{\mathbf{v}_{h} \in \mathbf{Z}_{h}}\left\|\mathbf{u}-\mathbf{v}_{h}\right\|_{h}+O s c(\mathbf{f}, \Omega)\right) \\
& +C \inf _{q_{h} \in Q_{h}}\left(\left\|p-q_{h}\right\|_{L^{2}(\Omega)}+\mathcal{S}_{h}\left(q_{h}, q_{h}\right)^{1 / 2}\right) .
\end{aligned}
$$

Proof. Let $\mathbf{v}_{h} \in \mathbf{Z}_{h}$ be arbitrary. Set $\mathbf{w}_{h}=\mathbf{u}_{h}-\mathbf{v}_{h}$. Then by using (3.5) and (3.3b),

$$
\begin{aligned}
C\left\|\mathbf{w}_{h}\right\|_{h}^{2} & \leq \mathcal{A}_{h}\left(\mathbf{u}_{h}, \mathbf{w}_{h}\right)-\mathcal{A}_{h}\left(\mathbf{v}_{h}, \mathbf{w}_{h}\right) \\
& =\mathbf{f}\left(\mathbf{w}_{h}\right)-\mathcal{B}_{h}\left(\mathbf{w}_{h}, p_{h}\right)-\mathcal{A}_{h}\left(\mathbf{v}_{h}, \mathbf{w}_{h}\right) \\
& =\mathbf{f}\left(\mathbf{w}_{h}\right)-\mathcal{B}_{h}\left(\mathbf{w}_{h}, q_{h}\right)-\mathcal{A}_{h}\left(\mathbf{v}_{h}, \mathbf{w}_{h}\right)-\mathcal{S}_{h}\left(p_{h}, p_{h}\right)+\mathcal{S}_{h}\left(p_{h}, q_{h}\right) .
\end{aligned}
$$

Therefore,

$$
\begin{aligned}
C\left\|\mathbf{w}_{h}\right\|_{h}^{2}+\mathcal{S}_{h}\left(p_{h}, p_{h}\right)= & \mathbf{f}\left(\mathbf{w}_{h}\right)-\mathcal{B}_{h}\left(\mathbf{w}_{h}, q_{h}\right)-\mathcal{A}_{h}\left(\mathbf{v}_{h}, \mathbf{w}_{h}\right)+\mathcal{S}_{h}\left(p_{h}, q_{h}\right) \\
= & \mathbf{f}\left(\mathbf{w}_{h}-E_{h} \mathbf{w}_{h}\right)-\mathcal{B}_{h}\left(\mathbf{w}_{h}-E_{h} \mathbf{w}_{h}, q_{h}\right)-\mathcal{A}_{h}\left(\mathbf{v}_{h}, \mathbf{w}_{h}-E_{h} \mathbf{w}_{h}\right) \\
& +\mathbf{f}\left(E_{h} \mathbf{w}_{h}\right)-\mathcal{B}_{h}\left(E_{h} \mathbf{w}_{h}, q_{h}\right)-\mathcal{A}_{h}\left(\mathbf{v}_{h}, E_{h} \mathbf{w}_{h}\right)+\mathcal{S}_{h}\left(p_{h}, q_{h}\right) \\
= & \mathbf{f}\left(\mathbf{w}_{h}-E_{h} \mathbf{w}_{h}\right)-\mathcal{B}_{h}\left(\mathbf{w}_{h}-E_{h} \mathbf{w}_{h}, q_{h}\right)-\mathcal{A}_{h}\left(\mathbf{v}_{h}, \mathbf{w}_{h}-E_{h} \mathbf{w}_{h}\right) \\
& +a\left(\mathbf{u}, E_{h} \mathbf{w}_{h}\right)+b\left(E_{h} \mathbf{w}_{h}, p\right)-\mathcal{B}_{h}\left(E_{h} \mathbf{w}_{h}, q_{h}\right)-\mathcal{A}_{h}\left(\mathbf{v}_{h}, E_{h} \mathbf{w}_{h}\right) \\
& +\mathcal{S}_{h}\left(p_{h}, q_{h}\right) .
\end{aligned}
$$

First note that

$$
\begin{aligned}
\left|a\left(\mathbf{u}, E_{h} \mathbf{w}_{h}\right)-\mathcal{A}_{h}\left(\mathbf{v}_{h}, E_{h} \mathbf{w}_{h}\right)\right| & \leq C\left\|\mathbf{u}-\mathbf{v}_{h}\right\|_{h}\left\|\mathbf{w}_{h}\right\|_{h} \\
\left|b\left(E_{h} \mathbf{w}_{h}, p\right)-\mathcal{B}_{h}\left(E_{h} \mathbf{w}_{h}, q_{h}\right)\right| & \leq C\left\|p-q_{h}\right\|_{L^{2}(\Omega)}\left\|\mathbf{w}_{h}\right\|_{h} \\
\left|\mathcal{S}_{h}\left(p_{h}, q_{h}\right)\right| & \leq \mathcal{S}_{h}\left(p_{h}, p_{h}\right)^{1 / 2} \mathcal{S}_{h}\left(q_{h}, q_{h}\right)^{1 / 2} .
\end{aligned}
$$


Using integration by parts,

$$
-\mathcal{B}_{h}\left(\mathbf{w}_{h}-E_{h} \mathbf{w}_{h}, q_{h}\right)=-\sum_{T \in \mathcal{T}_{h}} \int_{T} \nabla q_{h} \cdot\left(\mathbf{w}_{h}-E_{h} \mathbf{w}_{h}\right)+\sum_{e \in \mathcal{E}_{h}^{i}} \int_{e} \llbracket q_{h} \rrbracket \cdot\left\{\left\{\mathbf{w}_{h}-E_{h} \mathbf{w}_{h}\right\}\right\}
$$

and

$$
\begin{array}{r}
-\mathcal{A}_{h}\left(\mathbf{v}_{h}, \mathbf{w}_{h}-E_{h} \mathbf{w}_{h}\right)=\sum_{T \in \mathcal{T}_{h}} \int_{T} \Delta \mathbf{v}_{h} \cdot\left(\mathbf{w}_{h}-E_{h} \mathbf{w}_{h}\right)-\sum_{e \in \mathcal{E}_{h}^{i}} \int_{e} \llbracket \nabla_{h} \mathbf{v}_{h} \rrbracket \cdot\left\{\left\{\mathbf{w}_{h}-E_{h} \mathbf{w}_{h}\right\}\right\} \\
+\sum_{e \in \mathcal{E}_{h}} \int_{e}\left\{\left\{\nabla_{h}\left(\mathbf{w}_{h}-E_{h} \mathbf{w}_{h}\right)\right\}\right\}: \llbracket \underline{\mathbf{v}_{h} \rrbracket}-\sum_{e \in \mathcal{E}_{h}} \int_{e} \frac{\eta}{h_{e}} \llbracket \underline{\mathbf{v}_{h}} \rrbracket: \llbracket \underline{\mathbf{w}_{h}-E_{h} \mathbf{w}_{h} \rrbracket .}
\end{array}
$$

Hence,

$$
\begin{aligned}
\mathbf{f}\left(\mathbf{w}_{h}-E_{h} \mathbf{w}_{h}\right) & -\mathcal{B}_{h}\left(\mathbf{w}_{h}-E_{h} \mathbf{w}_{h}, q_{h}\right)-\mathcal{A}_{h}\left(\mathbf{v}_{h}, \mathbf{w}_{h}-E_{h} \mathbf{w}_{h}\right) \\
& =\sum_{T \in \mathcal{T}_{h}} \int_{T}\left(P \mathbf{f}+\Delta \mathbf{v}_{h}-\nabla q_{h}\right) \cdot\left(\mathbf{w}_{h}-E_{h} \mathbf{w}_{h}\right) \\
& -\sum_{e \in \mathcal{E}_{h}^{i}} \int_{e} \llbracket \nabla_{h} \mathbf{v}_{h}-q_{h} I \rrbracket \cdot\left\{\left\{\mathbf{w}_{h}-E_{h} \mathbf{w}_{h}\right\}\right\} \\
& +\sum_{e \in \mathcal{E}_{h}} \int_{e}\left\{\left\{\nabla_{h}\left(\mathbf{w}_{h}-E_{h} \mathbf{w}_{h}\right)\right\}\right\}: \llbracket \underline{\llbracket \mathbf{v}_{h} \rrbracket}-\sum_{e \in \mathcal{E}_{h}} \int_{e} \frac{\eta}{h_{e}} \llbracket \underline{\mathbf{v}_{h}} \rrbracket: \llbracket \underline{\left.\mathbf{w}_{h}-E_{h} \mathbf{w}_{h}\right] .} .
\end{aligned}
$$

By using Cauchy-Schwarz, inequality (2.1) and Lemma 2.2, we find

$$
\begin{aligned}
\mid \mathbf{f}\left(\mathbf{w}_{h}-E_{h} \mathbf{w}_{h}\right) & -\mathcal{B}_{h}\left(\mathbf{w}_{h}-E_{h} \mathbf{w}_{h}, q_{h}\right)-\mathcal{A}_{h}\left(\mathbf{v}_{h}, \mathbf{w}_{h}-E_{h} \mathbf{w}_{h}\right) \mid \\
& \leq C\left(\left\|\mathbf{u}-\mathbf{v}_{h}\right\|_{h}+\left\|p-q_{h}\right\|_{L^{2}(\Omega)}+O s c(\mathbf{f}, \Omega)\right)\left\|\mathbf{w}_{h}\right\|_{h} .
\end{aligned}
$$

Altogether, we complete the proof.

A few remarks are in order. In the equal order case we see that

$$
\begin{aligned}
\left\|\mathbf{u}-\mathbf{u}_{h}\right\|_{h}+\mathcal{S}_{h}\left(p_{h}, p_{h}\right)^{1 / 2} \leq & C\left(\inf _{\mathbf{v}_{h} \in \mathbf{Z}_{h}}\left\|\mathbf{u}-\mathbf{v}_{h}\right\|_{h}+O s c(\mathbf{f}, \Omega)\right) \\
& +C \inf _{q_{h} \in Q_{h} \cap H^{1}(\Omega)}\left\|p-q_{h}\right\|_{L^{2}(\Omega)} .
\end{aligned}
$$

Notice that in the last term we are taking the infimum over $Q_{h} \cap H^{1}(\Omega)$. In the case $Q_{h}=Q_{h}^{r-1}$ we can take the infimum over $Q_{h}$. In the next section we modify the method in the equal order case in order to improve this result.

In order to prove convergence of the DG methods under consideration we will have to argue that $O s c(\mathbf{f}, \Omega)$ approaches zero as $h$ tends to zero. We however, are not able to show this under the assumption that $\mathbf{f} \in\left[H^{-1}(\Omega) \cap L^{1}(\Omega)\right]^{d}$. Therefore, we prove convergence requiring more regularity on $\mathbf{f}$.

To this end, we establish the following inequality:

$$
O s c(\mathbf{f}, \Omega) \leq C\|\mathbf{f}-P \mathbf{f}\|_{H^{-1}(\Omega)} .
$$


A similar result was proved in [15]. Here we give an alternative proof for completeness. In order to prove (3.13), we recall the following Theorem [18]:

Theorem 3.2. For $\tilde{\mathbf{f}} \in\left[H^{-1}(\Omega)\right]^{d}$, there exists $\mathbf{F}=\left[F_{i j}\right] \in\left[L^{2}(\Omega)\right]^{d \times d}$ such that

$$
\tilde{\mathbf{f}}(\mathbf{v})=\int_{\Omega} \mathbf{F}: \nabla \mathbf{v} \quad \forall \mathbf{v} \in \mathbf{V}
$$

with

$$
\|\tilde{\mathbf{f}}\|_{H^{-1}(\Omega)}=\|\mathbf{F}\|_{L^{2}(\Omega)} .
$$

Proof. (of (3.13)) Let $\mathbf{v} \in H_{0}^{1}\left(\mathcal{T}_{e}\right)$ and let $\tilde{\mathbf{v}}$ be the extension of $\mathbf{v}$ by zero outside of $\mathcal{T}_{e}$. Using Theorem 3.2 with $\tilde{\mathbf{f}}=\mathbf{f}-P \mathbf{f}$,

$$
\left(\mathbf{f}_{e}-P \mathbf{f}\right)(\mathbf{v})=(\mathbf{f}-P \mathbf{f})(\tilde{\mathbf{v}})=\int_{\Omega} \mathbf{F}: \nabla \tilde{\mathbf{v}}=\int_{\mathcal{T}_{e}} \mathbf{F}: \nabla \mathbf{v} \quad \forall \mathbf{v} \in\left[H_{0}^{1}\left(\mathcal{T}_{e}\right)\right]^{d},
$$

we find

$$
\left\|\mathbf{f}_{e}-P \mathbf{f}\right\|_{H^{-1}\left(\mathcal{T}_{e}\right)}^{2} \leq\|\mathbf{F}\|_{L^{2}\left(\mathcal{T}_{e}\right)}^{2} .
$$

Therefore, the proof of (3.13) follows by summing over $e \in \mathcal{E}_{h}$ and using Theorem 3.2.

Below we prove that $\|\mathbf{f}-P \mathbf{f}\|_{H^{-1}(\Omega)} \rightarrow 0$ as $h \rightarrow 0$ whenever $\mathbf{f} \in\left[L^{p}(\Omega)\right]^{d}$ for $p>1$ if $d=2$, and $p \geq 6 / 5$ if $d=3$; these are exactly the assumptions made in [17].

First of all note that $\|P \mathbf{f}\|_{L^{p}(\Omega)} \leq C\|\mathbf{f}\|_{L^{p}(\Omega)}$. Then

$$
\|\mathbf{f}-P \mathbf{f}\|_{H^{-1}(\Omega)}=\sup _{\mathbf{v} \in\left[H_{0}^{1}(\Omega)\right]^{d}, \mathbf{v} \neq 0} \frac{(\mathbf{f}-P \mathbf{f})(\mathbf{v})}{\|\mathbf{v}\|_{H_{0}^{1}(\Omega)}}
$$

and

$$
\begin{aligned}
(\mathbf{f}-P \mathbf{f})(\mathbf{v})=(\mathbf{f}-P \mathbf{f})(\mathbf{v}-P \mathbf{v}) & \leq\|\mathbf{f}-P \mathbf{f}\|_{L^{p}(\Omega)}\|\mathbf{v}-P \mathbf{v}\|_{L^{q}(\Omega)} \\
& \leq C\|\mathbf{f}-P \mathbf{f}\|_{L^{p}(\Omega)} h^{1-d(1 / 2-1 / q)}\|\mathbf{v}\|_{H_{0}^{1}(\Omega)},
\end{aligned}
$$

where $p$ and $q$ are such that $1 / p+1 / q=1$. Therefore

$$
\|\mathbf{f}-P \mathbf{f}\|_{H^{-1}(\Omega)} \leq C h^{1-d(1 / 2-1 / q)}\|\mathbf{f}-P \mathbf{f}\|_{L^{p}(\Omega)} .
$$

Thus,

$$
O s c(\mathbf{f}, \Omega) \leq C h^{1-d(1 / 2-1 / q)}\|\mathbf{f}-P \mathbf{f}\|_{L^{p}(\Omega)} .
$$

The following corollary is a simple consequence of the previous results.

Corollary 3.3. Assume $\mathbf{f} \in\left[L^{p}(\Omega)\right]^{d}$ for $p>1$ if $d=2$, and $p>6 / 5$ when $d=3$. Then, $\left\|\mathbf{u}-\mathbf{u}_{h}\right\|_{h}+\mathcal{S}_{h}\left(p_{h}, p_{h}\right)^{1 / 2}$ converges to zero as $h$ approaches zero. Moreover, if we assume $[\mathbf{u}, p] \in H^{1+s}(\Omega) \times H^{s}(\Omega)$ for $0 \leq s \leq r$ and $\mathbf{f} \in W^{\ell, p}(\Omega)$ for an integer $0 \leq \ell \leq r+1$, then we have

$$
\left\|\mathbf{u}-\mathbf{u}_{h}\right\|_{h}+\mathcal{S}_{h}\left(p_{h}, p_{h}\right)^{1 / 2} \leq C h^{s}\left(\|\mathbf{u}\|_{H^{1+s}(\Omega)}+\|p\|_{H^{s}(\Omega)}\right)+C h^{1+\ell-d(1 / 2-1 / q)}\|\mathbf{f}\|_{W^{\ell, p}(\Omega)} .
$$


Proof. Using (3.12) and (3.14) we have

$$
\begin{aligned}
\left\|\mathbf{u}-\mathbf{u}_{h}\right\|_{h}+\mathcal{S}_{h}\left(p_{h}, p_{h}\right)^{1 / 2} \leq & C \inf _{\mathbf{v}_{h} \in \mathbf{Z}_{h}}\left\|\mathbf{u}-\mathbf{v}_{h}\right\|_{h} \\
& +C \inf _{q_{h} \in Q_{h}}\left(\left\|p-q_{h}\right\|_{L^{2}(\Omega)}+S_{h}\left(q_{h}, q_{h}\right)^{1 / 2}\right) \\
& +C h^{1-d(1 / 2-1 / q)}\|\mathbf{f}-P \mathbf{f}\|_{L^{p}(\Omega)} .
\end{aligned}
$$

The inequality (3.15) follows using approximation properties of $\mathbf{V}_{h}$ and $Q_{h}$ (or $Q_{h} \cap H_{0}^{1}$ when $\left.Q_{h}=Q_{h}^{r}\right)$

\section{Pressure Error Estimates}

Next, we derive the error estimate in the approximation of pressure.

Theorem 4.1. If $Q_{h}=Q_{h}^{r-1}$ it holds

$$
\left\|p-p_{h}\right\|_{L_{2}(\Omega)} \leq C\left(\inf _{\mathbf{v} \in \mathbf{Z}_{h}}\left\|\mathbf{u}-\mathbf{v}_{h}\right\|_{h}+\left\|p-P^{r-1} p\right\|_{L^{2}(\Omega)}+O s c(\mathbf{f}, \Omega)\right)
$$

and if $Q_{h}=Q_{h}^{r}$ it holds

$$
\begin{gathered}
\left\|p-p_{h}\right\|_{L_{2}(\Omega)} \leq C\left(\inf _{\mathbf{v} \in \mathbf{Z}_{h}}\left\|\mathbf{u}-\mathbf{v}_{h}\right\|_{h}+\left\|p-P^{r-1} p\right\|_{L^{2}(\Omega)}+O s c(\mathbf{f}, \Omega)\right) \\
+C \inf _{q_{h} \in Q_{h}}\left(\left\|p-q_{h}\right\|_{L^{2}(\Omega)}+\mathcal{S}_{h}\left(q_{h}, q_{h}\right)^{1 / 2}\right)
\end{gathered}
$$

where $P^{r-1}$ is the $L^{2}$ projection onto the space of piecewise polynomials of degree $r-1$.

Proof. Step 1: We first prove

$$
\left.\left\|P^{r-1}\left(p-p_{h}\right)\right\|_{L_{2}(\Omega)} \leq C\left(\left\|\mathbf{u}-\mathbf{u}_{h}\right\|_{h}+\left\|p-P^{r-1} p\right\|_{L^{2}(\Omega)}+O s c(\mathbf{f}, \Omega)\right)\right) .
$$

Note that this will prove the theorem in the case of $Q_{h}=Q_{h}^{r-1}$ (i.e. (4.1)).

To this end, let $m_{h}=P^{r-1} p$ and let $\theta_{h}=P^{r-1}\left(p-p_{h}\right)$. Then it is well-known (see for example [1]) that there exists $\mathbf{v} \in \mathbf{V}$ such that

$$
\begin{aligned}
\nabla \cdot \mathbf{v} & =\theta_{h} \quad \text { in } \Omega, \\
\|\mathbf{v}\|_{H^{1}(\Omega)} & \leq C\left\|\theta_{h}\right\|_{L_{2}(\Omega)} .
\end{aligned}
$$

Let $\Pi_{h}: \mathbf{V} \rightarrow \mathbf{V}_{h} \cap\left[H_{0}^{1}(\Omega)\right]^{d}$ be the Nedelec projection [22] defined by

$$
\begin{array}{ll}
\int_{T}\left(\mathbf{v}-\Pi_{h} \mathbf{v}\right) \cdot \mathbf{w}=0 & \forall \mathbf{w} \in \mathbf{N}^{r-1}(T) \\
\int_{e}\left(\mathbf{v}-\Pi_{h} \mathbf{v}\right) \cdot \mathbf{n} q=0 & \forall q \in \mathbb{P}^{r}(e), \forall e \subset \partial T
\end{array}
$$


where $\mathbf{N}^{r-1}(T)$ is the Nedelec space of index $r-1$. It is well known that the commuting property holds $\nabla \cdot \Pi_{h} \mathbf{v}=P^{r-1} \nabla \cdot \mathbf{v}[22]$ and that the following stability estimate holds

$$
\left\|\Pi_{h} \mathbf{v}\right\|_{h} \leq C\|\mathbf{v}\|_{H^{1}(\Omega)} \quad \text { for all } \mathbf{v} \in\left[H_{0}^{1}(\Omega)\right]^{d} .
$$

We also set $I_{h}:\left[H_{0}^{1}(\Omega)\right]^{d} \rightarrow \mathbf{V}_{h} \cap\left[H_{0}^{1}(\Omega)\right]^{d}$ to be the Scott-Zhang interpolant of degree $r$ [25]. We will also use the bound

$$
\left\|I_{h} \mathbf{v}\right\|_{H^{1}(\Omega)} \leq C\|\mathbf{v}\|_{H^{1}(\Omega)} \quad \text { for all } \mathbf{v} \in\left[H_{0}^{1}(\Omega)\right]^{d} .
$$

Then,

$$
\begin{aligned}
\left\|\theta_{h}\right\|_{L^{2}(\Omega)}^{2} & =\int_{\Omega}\left(\nabla \cdot \Pi_{h} \mathbf{v}\right) \theta_{h}=-\mathcal{B}_{h}\left(\Pi_{h} \mathbf{v}, \theta_{h}\right) \\
& =\mathcal{B}_{h}\left(\Pi_{h} \mathbf{v}, p_{h}\right)-\mathcal{B}_{h}\left(\Pi_{h} \mathbf{v}, m_{h}\right) \\
& =-\mathcal{A}_{h}\left(\mathbf{u}_{h}, \Pi_{h} \mathbf{v}\right)+\mathbf{f}\left(\Pi_{h} \mathbf{v}\right)-\mathcal{B}_{h}\left(\Pi_{h} \mathbf{v}, m_{h}\right) \\
& =-\mathcal{A}_{h}\left(\mathbf{u}_{h}, \Pi_{h} \mathbf{v}\right)+\mathbf{f}\left(\Pi_{h} \mathbf{v}\right)-\mathbf{f}\left(I_{h} \mathbf{v}\right)+a\left(\mathbf{u}, I_{h} \mathbf{v}\right)+b\left(I_{h} \mathbf{v}, p\right)-\mathcal{B}_{h}\left(\Pi_{h} \mathbf{v}, m_{h}\right) \\
& =-\mathcal{A}_{h}\left(\mathbf{u}_{h}, \Pi_{h} \mathbf{v}\right)+a\left(\mathbf{u}, I_{h} \mathbf{v}\right)-\mathbf{f}\left(I_{h} \mathbf{v}-\Pi_{h} \mathbf{v}\right)-\mathcal{B}_{h}\left(\Pi_{h} \mathbf{v}-I_{h} \mathbf{v}, m_{h}\right),
\end{aligned}
$$

where we used that $b\left(I_{h} \mathbf{v}, p-m_{h}\right)=0$ since $\nabla \cdot I_{h} \mathbf{v}$ is a piecewise polynomial of degree $r-1$. Using the definition of $\mathcal{A}_{h}$ we get

$$
\begin{aligned}
-\left\|\theta_{h}\right\|_{L^{2}(\Omega)}^{2}= & \sum_{T \in \mathcal{T}_{h}} \int_{T} \nabla \mathbf{u}_{h}: \nabla \Pi_{h} \mathbf{v}-\sum_{e \in \mathcal{E}_{h}} \int_{e}\left\{\left\{\nabla_{h} \mathbf{u}_{h}\right\}\right\}: \llbracket \underline{\Pi_{h} \mathbf{v} \rrbracket} \\
& -\sum_{e \in \mathcal{E}_{h}} \int_{e}\left\{\left\{\nabla_{h} \Pi_{h} \mathbf{v}\right\}\right\}: \llbracket \underline{\mathbf{u}_{h}} \rrbracket+\sum_{e \in \mathcal{E}_{h}} \int_{e} \frac{\eta}{h_{e}} \llbracket \underline{\mathbf{u}_{h}} \rrbracket: \llbracket \underline{\Pi \Pi_{h} \mathbf{v} \rrbracket} \\
& -a\left(\mathbf{u}, I_{h} \mathbf{v}\right)+\mathbf{f}\left(I_{h} \mathbf{v}-\Pi_{h} \mathbf{v}\right)+\mathcal{B}_{h}\left(\Pi_{h} \mathbf{v}-I_{h} \mathbf{v}, m_{h}\right) \\
= & \sum_{T \in \mathcal{T}_{h}} \int_{T} \nabla \mathbf{u}_{h}: \nabla\left(\Pi_{h} \mathbf{v}-I_{h} \mathbf{v}\right)-\sum_{e \in \mathcal{E}_{h}} \int_{e}\left\{\left\{\nabla_{h} \mathbf{u}_{h}\right\}\right\}: \llbracket \underline{\Pi_{h} \mathbf{v}-I_{h} \mathbf{v} \rrbracket} \\
& -\sum_{e \in \mathcal{E}_{h}} \int_{e}\left\{\left\{\nabla_{h} \Pi_{h} \mathbf{v}\right\}\right\}: \llbracket \underline{\mathbf{u}_{h}} \rrbracket+\sum_{e \in \mathcal{E}_{h}} \int_{e} \frac{\eta}{h_{e}} \llbracket \underline{\mathbf{u}_{h} \rrbracket}: \llbracket \underline{\Pi_{h} \mathbf{v}} \rrbracket \\
& +\left(\nabla_{h}\left(\mathbf{u}_{h}-\mathbf{u}\right), \nabla\left(I_{h} \mathbf{v}\right)\right)+\mathbf{f}\left(I_{h} \mathbf{v}-\Pi_{h} \mathbf{v}\right)+\mathcal{B}_{h}\left(\Pi_{h} \mathbf{v}-I_{h} \mathbf{v}, m_{h}\right) .
\end{aligned}
$$

Using integration by parts we see that

$$
\begin{aligned}
& \sum_{T \in \mathcal{T}_{h}} \int_{T} \nabla \mathbf{u}_{h}: \nabla\left(\Pi_{h} \mathbf{v}-I_{h} \mathbf{v}\right)-\sum_{e \in \mathcal{E}_{h}} \int_{e}\left\{\left\{\nabla_{h} \mathbf{u}_{h}\right\}\right\}: \llbracket \underline{\llbracket \Pi_{h} \mathbf{v}-I_{h} \mathbf{v} \rrbracket} \\
& +P \mathbf{f}\left(I_{h} \mathbf{v}-\Pi_{h} \mathbf{v}\right)+\mathcal{B}_{h}\left(\Pi_{h} \mathbf{v}-I_{h} \mathbf{v}, m_{h}\right) \\
& =-\sum_{T \in \mathcal{T}_{h}} \int_{T}\left(P \mathbf{f}+\Delta \mathbf{u}_{h}-\nabla m_{h}\right) \cdot\left(\Pi_{h} \mathbf{v}-I_{h} \mathbf{v}\right) d x
\end{aligned}
$$




$$
+\sum_{e \in \mathcal{E}_{h}^{i}} \int_{e} \llbracket \llbracket \nabla_{h} \mathbf{u}_{h}-m_{h} I \rrbracket \cdot\left\{\left\{I_{h} \mathbf{v}-\Pi_{h} \mathbf{v}\right\}\right\} d s
$$

Hence, we have

$$
\begin{aligned}
-\left\|\theta_{h}\right\|_{L^{2}(\Omega)}^{2}= & -\sum_{T \in \mathcal{T}_{h}} \int_{T}\left(P \mathbf{f}+\Delta \mathbf{u}_{h}-\nabla m_{h}\right) \cdot\left(\Pi_{h} \mathbf{v}-I_{h} \mathbf{v}\right) d x \\
& +\sum_{e \in \mathcal{E}_{h}^{i}} \int_{e} \llbracket \nabla_{h} \mathbf{u}_{h}-m_{h} I \rrbracket \cdot\left\{\left\{I_{h} \mathbf{v}-\Pi_{h} \mathbf{v}\right\}\right\} d s \\
& \left.-\sum_{e \in \mathcal{E}_{h}} \int_{e}\left\{\left\{\nabla_{h} \Pi_{h} \mathbf{v}\right\}\right\}: \llbracket \underline{\mathbf{u}_{h}} \rrbracket+\sum_{e \in \mathcal{E}_{h}} \int_{e} \frac{\eta}{h_{e}} \llbracket \underline{\mathbf{u}_{h}} \rrbracket: \llbracket \underline{\llbracket \Pi_{h} \mathbf{v}}\right] .
\end{aligned}
$$

Using inverse estimates, Lemma 2.2 and the bounds (4.8), (4.9), (4.5) we arrive at (4.3).

Step 2: We next prove that if $e \in \mathcal{E}_{h}$ and $e \subset T$ with $T \in \mathcal{T}_{h}$ then

$$
\begin{aligned}
h_{e}^{1 / 2}\left\|\left.\left(p_{h}-P^{r-1} p_{h}\right)\right|_{T}\right\|_{L^{2}(e)} \leq & C\left(\left\|\nabla\left(\mathbf{u}-\mathbf{u}_{h}\right)\right\|_{L^{2}(T)}+\frac{\eta}{h_{T}}\left\|\llbracket \underline{\mathbf{u}_{h}} \rrbracket\right\|_{L^{2}(\partial T)}\right) \\
& +C\left(\left\|p-P^{r-1} p\right\|_{L^{2}(T)}+\left\|\mathbf{f}_{e}-P \mathbf{f}\right\|_{H^{-1}\left(\mathcal{T}_{e}\right)}\right) \\
& +C\left(\left\|p-q_{h}\right\|_{L^{2}\left(\mathcal{T}_{e}\right)}+h_{e}^{1 / 2}\left\|\llbracket p_{h}-q_{h} \rrbracket\right\|_{L^{2}(e)}\right),
\end{aligned}
$$

where $q_{h} \in Q_{h}$ is arbitrary.

Fix an $e \in \mathcal{E}_{h}$ and find one $T \in \mathcal{T}_{h}$ be such that $e \subset \partial T$. We define $\mathbf{v} \in \mathbf{V}_{h}$ in the following way so that $\left.\mathbf{v}\right|_{\Omega \backslash T} \equiv 0$. Using the degrees of freedom of the Nedelec [22] space, define $\left.\mathbf{v}\right|_{T} \in\left[\mathbb{P}_{r}(T)\right]^{d}$ so that

$$
\begin{aligned}
\int_{e}\left(\mathbf{v} \cdot \mathbf{n}-\left(p_{h}-P^{r-1} p_{h}\right)\right) q & =0 \quad \text { for all } q \in \mathbb{P}_{r}(e) \\
\int_{e^{\prime}} \mathbf{v} \cdot \mathbf{n} q=0 & \text { for all } q \in \mathbb{P}_{r}\left(e^{\prime}\right) \text { for all edges } e^{\prime} \subset T \text { and } e^{\prime} \neq e \\
\int_{T} \mathbf{v} \cdot \mathbf{w}=0 & \text { for all } \mathbf{w} \in \mathbf{N}^{r-1}(T),
\end{aligned}
$$

where (by abuse of notation) in the integrations over $\partial T$ we consider the traces of the piecewise discontinuous functions taken from the interior of $T$.

A scaling argument gives

$$
\|\mathbf{v}\|_{L^{2}(T)} \leq C h_{T}^{1 / 2}\left\|p_{h}-P^{r-1} p_{h}\right\|_{L^{2}(e)},
$$

with the constant $C$ depending on $r$ but not on $h$. Note that

$$
\begin{aligned}
\mathcal{B}_{h}\left(p_{h}, \mathbf{v}\right) & =-\int_{T} p_{h} \nabla \cdot \mathbf{v}+\int_{e}\left\{\left\{p_{h}\right\}\right\} \mathbf{v} \cdot \mathbf{n} \\
& =-\int_{T} P^{r-1} p_{h} \nabla \cdot \mathbf{v}+\int_{e} p_{h} \mathbf{v} \cdot \mathbf{n}-\frac{1}{2} \int_{e} \llbracket p_{h} \rrbracket \cdot \mathbf{n}(\mathbf{v} \cdot \mathbf{n})
\end{aligned}
$$




$$
\begin{aligned}
& =\int_{T} \nabla\left(P^{r-1} p_{h}\right) \cdot \mathbf{v}+\int_{e}\left(p_{h}-P^{r-1} p_{h}\right) \mathbf{v} \cdot \mathbf{n}-\frac{1}{2} \int_{e} \llbracket p_{h} \rrbracket \cdot \mathbf{n}(\mathbf{v} \cdot \mathbf{n}) \\
& =\left\|p_{h}-P^{r-1} p_{h}\right\|_{L^{2}(e)}^{2}+\int_{T} \nabla\left(P^{r-1} p_{h}\right) \cdot \mathbf{v}-\frac{1}{2} \int_{e} \llbracket p_{h} \rrbracket \cdot \mathbf{n}(\mathbf{v} \cdot \mathbf{n}) .
\end{aligned}
$$

Therefore,

$$
\begin{aligned}
\left\|p_{h}-P^{r-1} p_{h}\right\|_{L^{2}(e)}^{2}= & -\mathcal{A}_{h}\left(\mathbf{u}_{h}, \mathbf{v}\right)+\mathbf{f}(\mathbf{v})-\int_{T} \nabla\left(P^{r-1} p_{h}\right) \cdot \mathbf{v}+\frac{1}{2} \int_{e} \llbracket p_{h} \rrbracket \cdot \mathbf{v} \\
= & \int_{T}\left(P \mathbf{f}+\Delta \mathbf{u}_{h}-\nabla\left(P^{r-1} p_{h}\right)\right) \cdot \mathbf{v}-\frac{1}{2} \int_{e} \llbracket \nabla_{h} \mathbf{u}_{h}-q_{h} I \rrbracket \cdot \mathbf{v} \\
& +\int_{\partial T}\left\{\left\{\nabla_{h} \mathbf{v}\right\}\right\}: \llbracket \underline{\mathbf{u}}_{h} \rrbracket-\sum_{g \subset \partial T} \int_{g} \frac{\eta}{h_{g}} \llbracket \underline{\mathbf{v}} \rrbracket: \llbracket \underline{\mathbf{u}_{h}} \rrbracket-\frac{1}{2} \int_{e} \llbracket p_{h}-q_{h} \rrbracket \cdot \mathbf{v},
\end{aligned}
$$

where $q_{h} \in Q_{h}$ is arbitrary. Applying inverse estimates we find

$$
\begin{gathered}
\left\|p_{h}-P^{r-1} p_{h}\right\|_{L^{2}(e)}^{2} \leq C\left(\left\|P \mathbf{f}+\Delta \mathbf{u}_{h}-\nabla\left(P^{r-1} p_{h}\right)\right\|_{L^{2}(T)}+h_{e}^{-1 / 2}\left\|\llbracket \nabla_{h} \mathbf{u}_{h}-q_{h} I \rrbracket\right\|_{L^{2}(e)}\right. \\
+\frac{\eta}{h_{T}^{3 / 2}}\left\|\llbracket \underline{\llbracket \mathbf{u}_{h}} \rrbracket\right\|_{L^{2}(\partial T)}+h_{e}^{-1 / 2}\left\|\llbracket\left[p_{h}-q_{h} \rrbracket\|\|_{L^{2}(e)}\right)\right\| \mathbf{v} \|_{L^{2}(T)} .
\end{gathered}
$$

Using (4.11) and Lemma 2.2 proves (4.10).

Step 3: Now we combine the previous two steps to finish the proof. By the triangle inequality we have

$$
\left\|p_{h}-p\right\|_{L^{2}(T)} \leq\left\|p-P^{r-1} p\right\|_{L^{2}(T)}+\left\|P^{r-1}\left(p-p_{h}\right)\right\|_{L^{2}(T)}+\left\|P^{r-1} p_{h}-p_{h}\right\|_{L^{2}(T)} .
$$

From a scaling argument we see that

$$
\left\|P^{r-1} p_{h}-p_{h}\right\|_{L^{2}(T)} \leq C h_{T}^{1 / 2}\left\|P^{r-1} p_{h}-p_{h}\right\|_{L^{2}(e)}
$$

for any edge $e$ of $T$, with the constant $C$ depending on $r$ but not on $h_{T}$. This follows from the fact that $P^{r-1} p_{h}-p_{h} \in \mathbb{P}_{r}(T)$ and that its moments up to degree $r-1$ vanish. If we use (4.3) and (4.10) we see that

$$
\begin{gathered}
\left\|p-p_{h}\right\|_{L_{2}(\Omega)} \leq C\left(\left\|\mathbf{u}-\mathbf{u}_{h}\right\|_{h}+S_{h}\left(p_{h}, p_{h}\right)^{1 / 2}+\left\|p-P^{r-1} p\right\|_{L^{2}(\Omega)}+O s c(\mathbf{f}, \Omega)\right) \\
+C \inf _{q_{h} \in Q_{h}}\left(\left\|p-q_{h}\right\|_{L^{2}(\Omega)}+\mathcal{S}_{h}\left(q_{h}, q_{h}\right)^{1 / 2}\right) .
\end{gathered}
$$

We arrive at (4.2) if we apply Theorem 3.1.

\section{Stabilizing With the Full Flux in the EQUal ORDer CASE}

We consider the equal order case $Q_{h}=Q_{h}^{r}$ and modify the method (3.3). Define $\left[\mathbf{u}_{h}, p_{h}\right] \in$ $\mathbf{V}_{h} \times Q_{h}$ as the solution to

$$
\mathcal{A}_{h}\left(\mathbf{u}_{h}, \mathbf{v}_{h}\right)+\mathcal{B}_{h}\left(\mathbf{v}_{h}, p_{h}\right)+\sum_{e \in \mathcal{E}_{h}^{i}} h_{e} \int_{e} \llbracket \nabla_{h} \mathbf{u}_{h}-p_{h} I \rrbracket \cdot \llbracket \nabla_{h} \mathbf{v}_{h} \rrbracket=\mathbf{f}\left(\mathbf{v}_{h}\right) \quad \forall \mathbf{v}_{h} \in \mathbf{V}_{h},
$$




$$
\mathcal{B}_{h}\left(\mathbf{u}_{h}, q_{h}\right)-\sum_{e \in \mathcal{E}_{h}^{i}} h_{e} \int_{e} \llbracket \nabla_{h} \mathbf{u}_{h}-p_{h} I \rrbracket \cdot \llbracket q_{h} I \rrbracket=0 \quad \forall q_{h} \in Q_{h} .
$$

Theorem 5.1. Consider the method defined by (5.1). It holds that

$$
\begin{aligned}
& \left\|\mathbf{u}-\mathbf{u}_{h}\right\|_{h}+\left(\sum_{e \in \mathcal{E}_{h}^{i}} h_{e} \int_{e} \llbracket \nabla_{h} \mathbf{u}_{h}-p_{h} I \rrbracket^{2}\right)^{1 / 2} \leq C\left(\inf _{\mathbf{v}_{h} \in \mathbf{Z}_{h}}\left\|\mathbf{u}-\mathbf{v}_{h}\right\|_{h}+\inf _{q_{h} \in Q_{h}}\left\|p-q_{h}\right\|_{L^{2}(\Omega)}\right) \\
& +C O s c(\mathbf{f}, \Omega) \text {. }
\end{aligned}
$$

Proof. If we let $\mathbf{w}_{h}=\mathbf{u}_{h}-\mathbf{v}_{h}$ for an arbitrary $\mathbf{v}_{h} \in \mathbf{Z}_{h}$ and follow similar arguments as the proof of Theorem 3.1 we arrive at

$$
\begin{aligned}
C\left\|\mathbf{w}_{h}\right\|_{h}^{2} & +\sum_{e \in \mathcal{E}_{h}^{i}} h_{e} \int_{e} \llbracket \nabla_{h} \mathbf{u}_{h}-p_{h} I \rrbracket^{2} \\
\leq & \mathbf{f}\left(\mathbf{w}_{h}-E_{h} \mathbf{w}_{h}\right)-\mathcal{B}_{h}\left(\mathbf{w}_{h}-E_{h} \mathbf{w}_{h}, q_{h}\right)-\mathcal{A}_{h}\left(\mathbf{v}_{h}, \mathbf{w}_{h}-E_{h} \mathbf{w}_{h}\right) \\
& +a\left(\mathbf{u}, E_{h} \mathbf{w}_{h}\right)+b\left(E_{h} \mathbf{w}_{h}, p\right)-\mathcal{B}_{h}\left(E_{h} \mathbf{w}_{h}, q_{h}\right)-\mathcal{A}_{h}\left(\mathbf{v}_{h}, E_{h} \mathbf{w}_{h}\right) \\
& +\sum_{e \in \mathcal{E}_{h}^{i}} h_{e} \int_{e} \llbracket \nabla_{h} \mathbf{u}_{h}-p_{h} I \rrbracket \cdot \llbracket \nabla_{h} \mathbf{v}_{h}-q_{h} I \rrbracket .
\end{aligned}
$$

Using the bounds in the proof of Theorem 3.1 (i.e. (3.6), (3.7) and (3.11)) we obtain

$$
\begin{aligned}
C\left\|\mathbf{w}_{h}\right\|_{h}^{2}+\sum_{e \in \mathcal{E}_{h}^{i}} h_{e} \int_{e} \llbracket \nabla_{h} \mathbf{u}_{h}-p_{h} I \rrbracket^{2} \leq C & \left(\left\|\mathbf{u}-\mathbf{v}_{h}\right\|_{h}+\left\|p-q_{h}\right\|_{L^{2}(\Omega)}+O s c(\mathbf{f}, \Omega)\right)\left\|\mathbf{w}_{h}\right\|_{h} \\
& +\sum_{e \in \mathcal{E}_{h}^{i}} h_{e} \int_{e} \llbracket \nabla_{h} \mathbf{v}_{h}-q_{h} I \rrbracket^{2} .
\end{aligned}
$$

We complete the proof after applying the second inequality of Lemma 2.2 and the triangle inequality.

The proof of the following pressure estimate follows a similar argument as in the proof of Theorem 4.1. We leave the details to the reader.

Theorem 5.2. Consider the method defined by (5.1). The following estimate holds:

$$
\left\|p-p_{h}\right\|_{L_{2}(\Omega)} \leq C\left(\inf _{\mathbf{v}_{h} \in \mathbf{Z}_{h}}\left\|\mathbf{u}-\mathbf{v}_{h}\right\|_{h}+\left\|p-P^{r-1} p\right\|_{L^{2}(\Omega)}+O s c(\mathbf{f}, \Omega)\right) .
$$

6. A DG METHOD FOR $\mathbf{f} \in\left[H^{-1}(\Omega)\right]^{d}$

It is clear that the DG method (3.3) is not well-defined for every $\mathbf{f} \in\left[H^{-1}(\Omega)\right]^{d}$. In order to have a well-defined method we modify the method in the following way

$$
\mathcal{A}_{h}\left(\mathbf{u}_{h}, \mathbf{v}_{h}\right)+\mathcal{B}_{h}\left(\mathbf{v}_{h}, p_{h}\right)=\mathbf{f}\left(E_{h} \mathbf{v}_{h}\right) \quad \forall \mathbf{v}_{h} \in \mathbf{V}_{h},
$$




$$
\mathcal{B}_{h}\left(\mathbf{u}_{h}, q_{h}\right)-\mathcal{S}_{h}\left(p_{h}, q_{h}\right)=0 \quad \forall q_{h} \in Q_{h},
$$

where we recall $E_{h}: V_{h} \rightarrow V_{h} \cap H_{0}^{1}(\Omega)$ satisfies (2.1). There are many choices for $E_{h}$ which are normally easy to compute.

We prove the following error estimate.

Theorem 6.1. Consider the method (6.1). There holds

$$
\begin{aligned}
\left\|\mathbf{u}-\mathbf{u}_{h}\right\|_{h}+\mathcal{S}_{h}\left(p_{h}, p_{h}\right)^{1 / 2} \leq & C \inf _{\mathbf{v}_{h} \in \mathbf{Z}_{h}}\left\|\mathbf{u}-\mathbf{v}_{h}\right\|_{h}+C\left(\sum_{e \in \mathcal{E}_{h}}\left\|\mathbf{f}_{e}\right\|_{H^{-1}\left(\mathcal{T}_{e}\right)}^{2}\right)^{1 / 2} \\
& +C \inf _{q_{h} \in Q_{h}}\left(\left\|p-q_{h}\right\|_{L^{2}(\Omega)}+\mathcal{S}_{h}\left(q_{h}, q_{h}\right)^{1 / 2}\right) .
\end{aligned}
$$

Notice that the difference in this estimate as compared to Theorem 3.1 is that $P \mathbf{f}$ does not appear. This will allow us to prove convergence assuming $\mathbf{f} \in\left[H^{-1}(\Omega)\right]^{d}$. Indeed, given $\epsilon>0$ there exists $\tilde{\mathbf{f}} \in\left[L^{2}(\Omega)\right]^{d}$ such that

$$
\|\mathbf{f}-\tilde{\mathbf{f}}\|_{H^{-1}(\Omega)} \leq \epsilon .
$$

Then,

$$
\begin{aligned}
\left(\sum_{e \in \mathcal{E}_{h}}\left\|\mathbf{f}_{e}\right\|_{H^{-1}\left(\mathcal{T}_{e}\right)}^{2}\right) & \leq\left(\sum_{e \in \mathcal{E}_{h}}\left\|\mathbf{f}_{e}-\tilde{\mathbf{f}}\right\|_{H^{-1}\left(\mathcal{T}_{e}\right)}^{2}\right)+\left(\sum_{e \in \mathcal{E}_{h}}\|\tilde{\mathbf{f}}\|_{H^{-1}\left(\mathcal{T}_{e}\right)}^{2}\right) \\
& \leq C\|\mathbf{f}-\tilde{\mathbf{f}}\|_{H^{-1}(\Omega)}^{2}+\left(\sum_{e \in \mathcal{E}_{h}}\|\tilde{\mathbf{f}}\|_{H^{-1}\left(\mathcal{T}_{e}\right)}^{2}\right)
\end{aligned}
$$

where we used (3.13). Using Poincare's inequality we have

$$
\|\tilde{\mathbf{f}}\|_{H^{-1}\left(\mathcal{T}_{e}\right)} \leq C h\|\tilde{\mathbf{f}}\|_{L^{2}\left(\mathcal{T}_{e}\right)}
$$

and hence we have

$$
\left(\sum_{e \in \mathcal{E}_{h}}\|\tilde{\mathbf{f}}\|_{H^{-1}\left(\mathcal{T}_{e}\right)}^{2}\right)^{1 / 2} \leq C h\|\tilde{\mathbf{f}}\|_{L^{2}(\Omega)}
$$

Therefore, we have

$$
\left(\sum_{e \in \mathcal{E}_{h}}\left\|\mathbf{f}_{e}\right\|_{H^{-1}\left(\mathcal{T}_{e}\right)}^{2}\right)^{1 / 2} \leq C\left(\epsilon+h\|\tilde{\mathbf{f}}\|_{L^{2}(\Omega)}\right) .
$$

There exists $h_{0}>0$ such that for $h<h_{0}$ we have $h\|\tilde{\mathbf{f}}\|_{L^{2}(\Omega)} \leq \epsilon$.

This shows that $\left(\sum_{e \in \mathcal{E}_{h}}\left\|\mathbf{f}_{e}\right\|_{H^{-1}\left(\mathcal{T}_{e}\right)}^{2}\right)^{1 / 2}$ converges to zero as $h$ approaches 0 . The other terms in Theorem 6.1 approach zero as $h$ approaches zero again using density arguments and therefore we can conclude that method (6.1) converges.

We now prove Theorem 6.1. 
Proof. (Theorem 6.1)

Following the proof of Theorem 3.1 we get

$$
\begin{aligned}
C\left\|\mathbf{w}_{h}\right\|_{h}^{2}+\mathcal{S}_{h}\left(p_{h}, p_{h}\right) \leq & -\mathcal{B}_{h}\left(\mathbf{w}_{h}-E_{h} \mathbf{w}_{h}, q_{h}\right)-\mathcal{A}_{h}\left(\mathbf{v}_{h}, \mathbf{w}_{h}-E_{h} \mathbf{w}_{h}\right) \\
& +a\left(\mathbf{u}, E_{h} \mathbf{w}_{h}\right)+b\left(E_{h} \mathbf{w}_{h}, p\right)-\mathcal{B}_{h}\left(E_{h} \mathbf{w}_{h}, q_{h}\right)-\mathcal{A}_{h}\left(\mathbf{v}_{h}, E_{h} \mathbf{w}_{h}\right) \\
& +\mathcal{S}_{h}\left(p_{h}, q_{h}\right) .
\end{aligned}
$$

Using (3.9) and (3.10) we have

$$
\begin{aligned}
- & \mathcal{B}_{h}\left(\mathbf{w}_{h}-E_{h} \mathbf{w}_{h}, q_{h}\right)-\mathcal{A}_{h}\left(\mathbf{v}_{h}, \mathbf{w}_{h}-E_{h} \mathbf{w}_{h}\right) \\
= & \sum_{T \in \mathcal{T}_{h}} \int_{T}\left(\Delta \mathbf{v}_{h}-\nabla q_{h}\right) \cdot\left(\mathbf{w}_{h}-E_{h} \mathbf{w}_{h}\right) \\
& -\sum_{e \in \mathcal{E}_{h}^{i}} \int_{e} \llbracket \nabla_{h} \mathbf{v}_{h}-q_{h} I \rrbracket \cdot\left\{\left\{\mathbf{w}_{h}-E_{h} \mathbf{w}_{h}\right\}\right\} \\
& +\sum_{e \in \mathcal{E}_{h}} \int_{e}\left\{\left\{\nabla_{h}\left(\mathbf{w}_{h}-E_{h} \mathbf{w}_{h}\right)\right\}\right\}: \llbracket \mathbf{v}_{h} \rrbracket-\sum_{e \in \mathcal{E}_{h}} \int_{e} \frac{\eta}{h_{e}} \llbracket\left[\mathbf{v}_{h} \rrbracket: \llbracket \mathbf{w}_{h}-E_{h} \mathbf{w}_{h} \rrbracket .\right.
\end{aligned}
$$

The proof is completed by applying Lemma 2.2 with $\mathbf{f}_{h}=0,(3.6)$ and (3.7).

Notice that Theorem 6.1 only gives at most an $O(h)$ convergence rate, even if $\mathbf{f}$ is smooth due to the term $\left(\sum_{e \in \mathcal{E}_{h}}\left\|\mathbf{f}_{e}\right\|_{H^{-1}\left(\mathcal{T}_{e}\right)}^{2}\right)^{1 / 2}$.

In order to remove this term altogether one can use instead an enrichment operator $E_{h}$ : $\mathbf{V}_{h} \rightarrow \mathbf{V}_{h}^{s} \cap\left[H_{0}^{1}(\Omega)\right]^{d}$ where

$$
\mathbf{V}_{h}^{s}=\left\{\mathbf{v}_{h} \in\left[L^{2}(\Omega)\right]^{d}:\left.\mathbf{v}_{h}\right|_{T} \in\left[\mathbb{P}_{s}(T)\right]^{d}\right\},
$$

with $s>r$. For example, suppose $Q_{h}=Q_{h}^{r-1}$ and suppose $d=2$. Then, we can define $E_{h}: \mathbf{V}_{h} \rightarrow \mathbf{V}_{h}^{r+1} \cap\left[H_{0}^{1}(\Omega)\right]^{d}$ in the following way

$$
\begin{aligned}
& \int_{T}\left(E_{h} \mathbf{w}_{h}-\mathbf{w}_{h}\right) \cdot \mathbf{v}=0 \quad \text { for all } \mathbf{v} \in\left[\mathbb{P}_{r-2}(T)\right]^{d} \text { for all } T \in \mathcal{T}_{h} \\
& \int_{e}\left(E_{h} \mathbf{w}_{h}-\left\{\left\{\mathbf{w}_{h}\right\}\right\}\right) \cdot \mathbf{v}=0 \quad \text { for all } \mathbf{v} \in\left[\mathbb{P}_{r-1}(e)\right]^{d} \text { for all edges } e \subset \mathcal{E}_{h}^{i} \\
& E_{h} \mathbf{w}_{h}(z)=\left.\frac{1}{\left|\mathcal{T}_{z}\right|} \sum_{T \in \mathcal{T}_{z}} \mathbf{w}_{h}\right|_{T}(z) \quad \text { for all vertices } z \in \mathcal{V}_{h}^{i} \\
& \int_{e} E_{h} \mathbf{w}_{h} \cdot \mathbf{v}=0 \quad \text { for all } \mathbf{v} \in\left[\mathbb{P}_{r-1}(e)\right]^{d} \text { for all edges } e \in \mathcal{E}_{h}^{b} \\
& E_{h} \mathbf{w}_{h}(z)=0 \quad \text { for all vertices } z \in \mathcal{V}_{h}^{b},
\end{aligned}
$$

where $\left|\mathcal{T}_{z}\right|$ denotes the cardinality of the set $\mathcal{T}_{z}$ and $\mathbb{P}_{-1}(T)=\{0\}$ for all $T \in \mathcal{T}_{h}$. Also not difficult to see that $E_{h}$ also satisfies (2.1). 
In this case, we have

$$
\begin{aligned}
& -\mathcal{B}_{h}\left(\mathbf{w}_{h}-E_{h} \mathbf{w}_{h}, q_{h}\right)-\mathcal{A}_{h}\left(\mathbf{v}_{h}, \mathbf{w}_{h}-E_{h} \mathbf{w}_{h}\right) \\
& =\sum_{e \in \mathcal{E}_{h}} \int_{e}\left\{\left\{\nabla_{h}\left(\mathbf{w}_{h}-E_{h} \mathbf{w}_{h}\right)\right\}\right\}: \llbracket \mathbf{v}_{h} \rrbracket-\sum_{e \in \mathcal{E}_{h}} \int_{e} \frac{\eta}{h_{e}} \llbracket \mathbf{v}_{h} \rrbracket: \llbracket \mathbf{w}_{h}-E_{h} \mathbf{w}_{h} \rrbracket .
\end{aligned}
$$

Hence, following the proof of Theorem 6.1 we get the following estimate

$$
\left\|\mathbf{u}-\mathbf{u}_{h}\right\|_{h} \leq C\left(\inf _{\mathbf{v}_{h} \in \mathbf{Z}_{h}}\left\|\mathbf{u}-\mathbf{v}_{h}\right\|_{h}+\inf _{q_{h} \in Q_{h}}\left\|p-q_{h}\right\|_{L^{2}(\Omega)}\right) .
$$

This shows that best approximation error estimates (mod a constant) are obtained for DG methods (6.1) by choosing the enrichment operator $E_{h}$ carefully.

Acknowledgements: The third and fourth authors are grateful to the Indo-US Virtual Institute of Mathematical and Statistical Sciences for sponsoring short term visit. The work of the first author has been funded by the European Research Council under the FP7 Programme Ideas through the Starting Grant No. 258443 - COMFUS: Computational Methods for Fusion Technology.

\section{REFERENCES}

[1] G. Acosta, R. Durán and M.A. Muschietti, Solutions of the divergence operator on John domains, Adv. Math. 206 (2006), no. 2, 373-401.

[2] M. Ainsworth and J. T. Oden. A posteriori error estimation in finite element analysis. Pure and Applied Mathematics (New York). Wiley-Interscience [John Wiley \& Sons], New York, 2000.

[3] D. N. Arnold, F. Brezzi, B. Cockburn and L. D. Marini. Unified analysis of discontinuous Galerkin methods for elliptic problems. SIAM J. Numer. Anal., 39:1749-1779, 2002.

[4] G. A. Baker, W. N. Jureidini and O. A. Karakashian. Piecewise Solenoidal vector fields and the Stokes problem. SIAM J. Numer. Anal. , 27:1466-1485, 1990.

[5] A. Berger, R. Scott and G. Strang. Approximate boundary conditions in the finite element method. In : Symposia Mathematica, Vol. X (Convegno di Analasi Numerica), London : Academic Press 1972, 295-313, 1972.

[6] D. Boffi, F. Brezzi, and M. Fortin. Finite elements for the Stokes problem. In : Mixed finite elements, compatibility conditions, and applications, Lectures given at the C.I.M.E. Summer School held in Cetraro, Italy, June 26-July 1, 2006. Lecture Notes in Mathematics. Springer Verlag. Vol. 1939 (2008), pp. 45-100. D. Boffi, L. Gastaldi editors.

[7] S.C. Brenner. Two-level additive Schwarz preconditioners for nonconforming finite element methods. Math. Comp., 65:897-921, 1996.

[8] S.C. Brenner. Convergence of nonconforming multigrid methods without full elliptic regularity. Math. Comp., 68:25-53, 1999.

[9] S.C. Brenner. Ponicaré-Friedrichs inequalities for piecewise $H^{1}$ functions. SIAM J. Numer. Anal, 41:306324, 2003.

[10] S.C. Brenner and L.R. Scott. The Mathematical Theory of Finite Element Methods (Third Edition). Springer-Verlag, New York, 2008.

[11] P.G. Ciarlet. The Finite Element Method for Elliptic Problems. North-Holland, Amsterdam, 1978.

[12] B. Cockburn, G. Kanschat, D. Schötzau and C. Schwab. Local discontinuous Galerkin methods for the Stokes system. SIAM J. Numer. Anal., 40:319-343, 2002. 
[13] R. Codina and S. Badia On the design of discontinuous Galerkin methods for elliptic problems based on hybrid formulations. Preprint.

[14] R. Codina, J. Principe and J. Baiges. Subscales on the element boundaries in the variational two-scale finite element method Comput. Methods Appl. Mech. Engrg., 198:838-852, 2009.

[15] A. Cohen, R. Devore and R.H. Nochetto, Convergence rates for AFEM with H-1 data. Preprint.

[16] M. Crouzeix and P. A. Raviart. Conforming and Nonconforming finite element methods for solving the stationary Stokes equations. RAIRO 7, rev. 3:33-76, 1973.

[17] D.A. Di Pietro and A. Ern. Discrete functional analysis tools for discontinuous Galerkin methods with application to the incompressible Navier-Stokes equations Math. Comp., 79: 1303-1330. 2010.

[18] P. Grisvard. Elliptic Problems in Nonsmooth Domains. Pitman, Boston, 1985.

[19] T. Gudi. A new error analysis for discontinuous finite element methods for linear elliptic problems. Math. Comp., 79: 2169-2189, 2010.

[20] T. Gudi. Some nonstandard error analysis of discontinuous Galerkin methods for elliptic problems. Calcolo, 47:239-261, 2010.

[21] P. Hansbo and M.G. Larson. Discontinuous Galerkin methods for incompressible and nearly incompressible elasticity by Nitsche's method. Comput. Methods Appl. Mech. Engrg., 191:1895-1908, 2002.

[22] J.-C. Nedelec, Mixed Finite Elements in $\mathbb{R}^{3}$, Numer. Math. 35(1980), 315-341.

[23] B. Rivière and T.P. Wihler Discontinuous Galerkin methods for second-order elliptic PDE with lowregularity solutions. J. Sci. Comput., 46:151-165, 2011.

[24] D. Schötzau, C.Schwab and A. Tosselli. Mixed hp-DGFEM for incompressible flows. SIAM J. Numer. Anal. , 40:2171-2194, 2003.

[25] R. Scott and S. Zhang, Finite Element interpolation of nonsmooth functions satisfying boundary conditions, Math. Comp., 54 (1990), 483-493.

[26] R. Verfürth. A posteriori error estimation and adaptive mesh-refinement techniques. In Proceedings of the Fifth International Congress on Computational and Applied Mathematics (Leuven, 1992), volume 50, pages $67-83,1994$.

[27] R. Verfürth. A Review of A Posteriori Error Estmation and Adaptive Mesh-Refinement Techniques. Wiley-Teubner, Chichester, 1995.

International Center for Numerical Methods in Engineering (Cimne), Universitat Polit'ecnica de Catalunya, Jordi Girona 1-3, Edifici C1, 08034 Barcelona, Spain

E-mail address: sbadia@cimne.upc.edu

Universtitat Politecnica de Catalunya (UPC), Jordi Girona 1-3, 08034 Barcelona, Spain

E-mail address: ramon.codina@upc.edu

Department of Mathematics, Indian Institute of Science, Bangalore 56002

E-mail address: gudi@math.iisc.ernet.in

Division of Applied Mathematics, Brown University, Providence, RI 02912

E-mail address: Johnny_Guzman@brown.edu 